\title{
Subjective uncertainty, the orientation reaction, and the reinforcement of an instrumental response ${ }^{1}$
}

\author{
R. M. NICKI ${ }^{2}$ AND J. F. SHEA ${ }^{3}$ \\ UNIVERSITY OF NEW BRUNSWICK
}

In Experiment 1, Ss were exposed to slides of intermediate blur for 5, 10,30, or $60 \mathrm{sec}$ and were asked to guess the identity of the blurred object and to estimate how confident they were of each guess. The $S$ 's task in Experiment 2 was merely to view the blurred slides while $S$ 's EEG waves were being recorded. After being exposed to a blurred slide for a certain duration, in Experiment 3, Ss were required, by means of a key press, to choose to view either a clear version of the blurred slide or an unrelated clear picture. Uncertainty/second, EEG desynchronization/second, and related choices were all found to be a negatively sloped function of viewing duration.

Recent experiments (Berlyne \& Borsa, 1968) have provided evidence that slides blurred to an intermediate degree evoked longer EEG desynchronization than did their clear counterparts when the slides wete presented in the sequence, blurred slide-clear slide, but not in the sequence, clear slide-blurred slide. Previously, Nicki (1967) had shown that subjective uncertainty (SU), equated to the average information formula, where $\mathbf{N}$ was the number of guesses as to the identity of the blurred object and $p_{i}$ was the relative certainty of each guess, was an inverted $U$-shaped function of the degree of blurredness. Furthermore, this result was used to accurately predict that in a two-choice situation, key presses that obtained a clear version of a blurred slide rather than an unrelated clear picture would be a similar function of blurredness. That SU and not blurredness alone was necessary to achieve this effect was affirmed by the finding that when subjective uncertainty was removed by presenting a clear version of the blurred object prior to the presentation of the blurred object, the effect vanished.

However, among the above studies, the viewing time of the blurred slides varied. For Berlyne and Borsa (1968), it was $4 \mathrm{sec}$, for Nicki's (1967) guessing situation, $30 \mathrm{sec}$, and for the choice situation, $10 \mathrm{sec}$. On the formal level, two properties of SU are shared by the concept, conflict (see Berlyne, 1957); on the empirical level, a blurred slide may be looked upon as evoking competing response tendencies as to the object's identity. Furthermore, one component of the orientation reaction, GSR, has been shown to be intensified by conflict (Berlyne, 1961). Factors operating over time, such as habituation and extinction, would affect the strength of the competing response tendencies and, therefore, conflict or SU. The three experiments reported investigated the relation between viewing time of the blurred object and SU, related key presses, and EEG desynchronization, respectively.

\section{EXPERIMENT 1}

Subjects

The Ss were 24 female undergraduates taking the introductory course in psychology.

\section{Apparatus}

The materials consisted of a slide projector (Kodak Carousel, Model 550, 5-in., f/3.5 lens), a projection screen, a stopwatch, and 20 medium-blurred, black-and-white slides of common objects comparable to those used by Nicki (1967) and Berlyne and Borsa (1968). There were four groups of durations; the particular group presented to $S$ was randomly determined. The sequence of durations for one group was determined by randomizing each set of four durations, $5,10,30$, and $60 \mathrm{sec}$. A Lat.n-square arrangemen: provided the sequence of durations for the other groups.

\section{Procedure}

The $S$ was seated at a small table facing the screen; E was seated to S's left. The S was given the following instructions: "This is a perception experiment. On that screen, you'll see a series of blurred slides. Each blurred slide will be presented for 5,10 , 30 , or $60 \mathrm{sec}$. Your job in this experiment is to guess what the object in each slide is, i.e., name it. However, it is not important whether your guesses are right or wrong. What is important is that you tell me all the guesses that come to mind while the slide is on the screen. If you have more guesses than you can say during the presentation of the blurred slide, keep guessing. I will replace the slide with a blank one while you continue. To repeat, I don't want you to mull over the possibilities in your mind and then come out with the best one. Instead, as soon as the slide comes on the screen, give me all the guesses that come to mind as to what the blurred object is."

After the first slide was on the screen for $5,10,30$, or $60 \mathrm{sec}$, a blank slide appeared, and $S$ was told, after she stopped guessing, that: "There's another thing I want you to do in this experiment. I want you to tell me how certain you are as to whether or not each of your guesses is correct. That is, 1 want you to give me a number ranging from $0 \%$ to $100 \%$ for each of your guesses. $0 \%$ represents complete uncertainty; $100 \%$ represents complete certainty. OK?"

The blurred slide reappeared on the screen. The E repeated each of S's guesses, and $S$ provided certainty estimates. The Ss were cautioned not to free-associate or to describe the object. Most Ss did not have difficulty with the task.

\section{Results and Discussion}

The SU values were calculated for each slide. Each per cent estimate was identified as $p_{i}$, normalized by dividing each $p_{i}$ value by

$$
\sum_{i}^{N} p_{i} \text { in order that } \underset{i}{\stackrel{N}{N}} p_{i}=1
$$

-a primary informational requisite, and inserted into the average information formula. Total SU values for each duration and for each $S$ were then divided by the length of duration in seconds. Inspection of Fig. 1 indicates that SU/second is a negatively sloped function of blurredness. An analysis of variance indicated the main effect of duration to be significant $[F(3,69)=18.00, p<.01]$. In addition, a trend analysis revealed a significant linear component $[F(1,69)=45.00, p<.01]$ and a significant quadratic component $[F(1,69)=8.00, p<.01]$. The results seemed reasonable in that one would expect the number of guesses to diminish over time, and also, perhaps, that later guesses might be deemed less certain than earlier ones, creating a larger discrepancy among the $\mathrm{p}_{i}$ values (SU depends on the relative, not absolute, value of the $p_{i} s$ ).

\section{Subjects}

\section{EXPERIMENT 2}

The Ss were 56 male undergraduates 

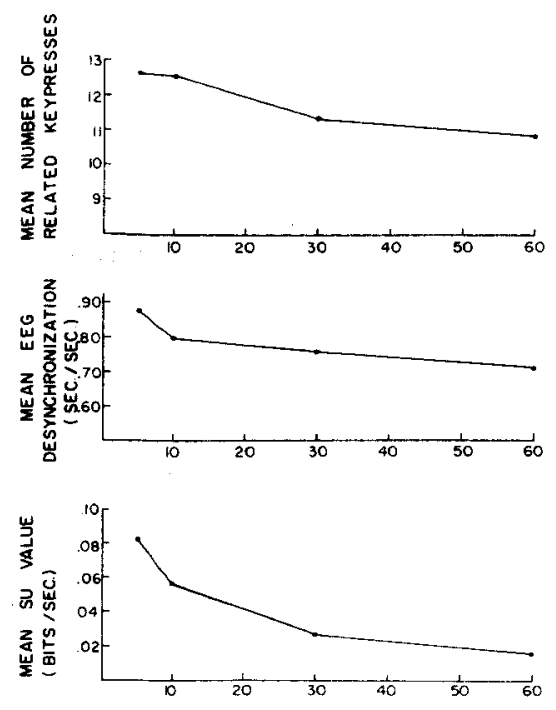

DURATION (SEC)

Fig. 1. The relation between subjective uncertaint y/second, EEG desynchronization/second (blurred slides, blurred-clear order), related choices, and viewing duration.

enrolled in the introductory course in psychology.

\section{Apparatus}

The materials consisted of the slide projector, and 16 of the medium-blurred slides of Experiment 1 and their corresponding clear versions; a stopwatch; a projection screen; Harvard Apparatus Co. amplifier (Model 355), event time marker (Model 281), recorder (Model 350), and chart mover (Model 485); and three bipotential skin electrodes (Beckman Instruments Co.) attached to S's left and right occipital lobes and the nape of the neck, using electrolyte gel and disposable adhesive collars. The blurred and clear slides were matched to form 16 pairs, and 4 pairs were randomly assigned to each of four sets. There were four different durations in each set, $5,10,30$, or $60 \mathrm{sec}$, the sequence of each being randomly determined. After the sequence of durations was arrived at for one group of 16 slides, three other groups were formed using a Latin-square arrangement. The particular group viewed by $S$ was randomly determined. For half of the Ss, the order of slides for the first set was blurred-clear; second set, clear-blurred; third set, blurred-clear; fourth set, clear-blurred. The opposite set orders prevailed for the other Ss. For any given duration, e.g., $5 \mathrm{sec}$, the blurred slide and the clear slide were each on the screen for $5 \mathrm{sec}$. Each slide was followed by a blank slide.
Procedure

The $S$ was seated in a recliner chair facing the screen $10 \mathrm{ft}$ in front of him. The $E$ sat behind $S$ near the projector. The $S$ was told that the aim of the study was to record his brain activity while he looked at a series of blurred and clear slides, and that he should relax as much as possible. In addition, $\mathbf{S}$ was informed that the slides would be presented in matched pairs for the durations specified above; before the presentation of each set of four pairs, $S$ was informed of the particular order to follow. Recording techniques, criteria for discarding Ss, and methods for scoring responses were very similar to those used by Berlyne and Nicki (1966) and by Berlyne and McDonnell (1965).

\section{Results and Discussion}

The amount of EEG desynchronization/second for blurred slides in the order, blurred-clear, was plotted in Fig. 1, revealing a negatively sloped curve. The effect of duration was found to be significant $[F(3,165)=23.27$, $\mathrm{p}<.01]$. A trend analysis indicated significant linear $[F(1,165)=56.52$, $\mathrm{p}<.001]$, quadratic $[\mathrm{F}(1,165)=5.99$, $\mathrm{p}<.025]$, and cubic components $[F(1,165)=7.32, \quad p<.01]$. Thus, a similarity is to be seen between the function relating $\mathrm{SU} /$ second to viewing duration and EEG desynchronization and duration.

An analysis of variance of the rate data for the entire experiment revealed a significant condition effect $[F(3,495)=6.88, p<.01]$, i.e., there were four conditions, blurred first $\left(\mathrm{Bl}_{1}\right)$, blurred second $\left(\mathrm{Bl}_{2}\right)$, clear first $\left(\mathrm{Cl}_{1}\right)$, and clear second $\left(\mathrm{Cl}_{2}\right)$. A linear comparison of $\mathrm{Bl}_{1}$ vs $\mathrm{Bl}_{2}, \mathrm{Cl}_{1}$, and $\mathrm{Cl}_{2}$ indicated a significant difference $[F(1,495)=18.71, p=.01]$. This finding provided further support for the conclusion that blurred pictures when associated with SU evoke longer desynchronization than do clear pictures (see Berlyne \& Borsa, 1968).

\section{EXPERIMENT 3}

Subjects

The Ss were 80 female students enrolled in the introductory psychology course.

\section{Apparatus}

In S's room were three slide projectors (Kodak Carousel, Model 55, 5-in., f/3.5), each with a rapid-action shutter placed in front of the lens; programming equipment; a door-buzzer on the floor to S's right; two telegraph keys mounted on a black board placed on a small table in front of $S$; the 20 blurred slides of Experiment 1, 20 blurred slides comparable to these, and 40 clear versions of the blurred slides. The $\mathbf{4 0}$ clear slides were assigned randomly to two slide trays, $\mathrm{Cl}_{\mathbf{a}}$ and $\mathrm{Cl}_{\mathbf{b}}$; correspondingly, there were two trays, $\mathrm{Bl}_{\mathrm{a}}$ and $\mathrm{Bl}_{\mathrm{b}}$, containing their blurred counterparts. For a given $S$, the three slide projectors contained Trays $\mathrm{Bl}_{\mathrm{a}}, \mathrm{Cl}_{\mathrm{a}}$, and $\mathrm{Cl}_{\mathrm{b}}$ or $\mathrm{Bl}_{\mathrm{b}}, \mathrm{Cl}_{\mathrm{a}}$, and $\mathrm{Cl}_{\mathrm{b}}$. Whether $\mathrm{S}$ received the former group or the latter and whether the left or right key obtained $\mathrm{Cl}_{\mathbf{a}}$ or $\mathrm{Cl}_{\mathbf{b}}$ was randomly determined.

\section{Procedure}

Each $S$ was seated and faced the screen about $10 \mathrm{ft}$ in front of her. The projectors were behind $S$. There were 20 trials in all for each $S$. On each trial, $S$ was presented a blurred slide for a certain duration; the buzzer then briefly sounded. By pressing one of the two telegraph keys once, $S$ obtained either a clear slide related to the blurred slide or a clear slide unrelated to the blurred slide for 5 sec. After this 5 -sec period, the next blurred slide automatically came on the screen. Throughout the experiment, one key was always associated with the related clear slide.

As in Nicki's (1967) experiment, $S$ was simply instructed that she would be looking at some slides, and that when she heard a buzzer, she was to press one of the keys in front of her. While she was not pressing, she was told to place her hand midway between the keys.

There were four groups of $20 \mathrm{Ss}$. Each group received one of the durations, 5,10 , 30 , or $60 \mathrm{sec}$, used in the previous experiment. The $E$ left the room after the instructions were given.

\section{Results and Viscussion}

In Fig. 1, it is observed that the number of related key presses is a negatively sloped function of duration. The effect of duration was significant $[F(3,57)=4.94$, $\mathrm{p}<.025]$. An analysis of trend indicated a significant linear component $[F(1,57)=5.97, p<.025]$ and $a$ significant cubic component $[\mathrm{F}(1,57)=8.05, \mathrm{p}<.025]$. Thus, the function relating related key presses to duration is similar to that relating EEG desynchronization/second and SU/second to duration. Wilcoxon tests indicated that the number of related key presses for three groups was significantly greater than $\mathbf{1 0 . 0 0}$ (5-sec group, $\mathrm{T}(19)=15, \mathrm{p}<.01 ; 10-\mathrm{sec}$ group, $T(17)=11, p<.01 ; 30-\sec$ group, $\mathrm{T}(17)=26, \mathrm{p}<.01 ; 60-\mathrm{sec}$ group, $T(15)=44, p<.05)$. In addition, the data were grouped into four blocks of five trials. An analysis of trend indicated only a significant overall linear component $[F(1,228)=38.11, p<.01]$. The Duration by Trials interaction was nonsignificant $[F(9,228)=1.01]$ as well as components of this interaction.

The fact that the mean number of 
related key presses was greater than 10.00 for three durations attests to the reinforcing aspect of $S U$ reduction and replicates Nick's' (1967) finding with only a 10-sec group. That the number of related key presses increases over time indicates that $S U$ reduction reinforces a response in a manner similar to that of hunger or thirst reduction. The nonsignificant overall quadratic component of trend might be rashly interpreted as contradicting this statement. However, this would be an erroneous conclusion, since SU is hypothesized to be newly aroused on each trial.

The similar function found in all three experiments further implicates $\mathrm{SU}$ as an energizer of behavior. That is, an instrumental response, a key press, is energized to the extent of SU or Beta activity present at the time of the key press. Energization of responses by SU decreases as viewing duration increases as a result of the extinction of the response of hypothesizing or guessing as to the identity of the blurred object by $S$, since hypothesizing does not bring about SU reduction. As the extinction of incompatible guessing occurs over time, conflict, as well as the orientation reaction, decreases.

\section{REFERENCES}

BERLYNE, D. E. Uncertainty and conflict: A point of contact between information theory and behavior theory. Psychological Review, 1957, 64, 329-339.

BERLYNE, D. E. Conflict and the orientation reaction. Journal of Experimental Psychology, $1961,62,476.483$.

BERLYNE, D. E., \& BORSA, D. M. Uncertainty and the orientation reaction. Perception \& Psychophysics, 1968, 3, 77-79.

BERLYNE, D. E., \& MCDONNELL, P. Effects of stimulus complexity and incongruity on duration of EEG desynchronization. Electroencephalography \& Clinical Neurophysiology, 1965, 18, 156-161.

BERLYNE, D. E., \& NICKI, R. M. Effects of pitch and duration of tones on EEG desynchronization. Psychonomic Science, 1966, 4, 101-102.

NICKI, R. M. The reinforcing effect of uncertainty reduction on a human operant. Thesis submitted in accordance with requirements for $\mathrm{PhD}$ degree, University of Toronto, 1967.

\section{NOTES}

1. This project was supported by Research Grant APA-262 from the National Research Council of Canada.

2. Address: The University of New Brunswick, Fredericton, New Brunswick, Canada.

3. The authors are indebted to Mrs. Jean Miller who carried out the experiments and helped in the analysis of the data.

(Accepted for publication August 27, 1969.) 